\title{
Evaluation of the Quality of Life of Anaesthesiology Residents and Attendings in Portugal
}

\author{
J. Pedreira ${ }^{1}, \underline{\text { C. Lanzaro }}{ }^{1}$, L. Ferreira ${ }^{1}$, A. Bernardino ${ }^{1}$, M. L. Bela ${ }^{1}$ \\ 1. Centro Hospitalar e Universitário de Coimbra, Portugal
}

\section{Background}

In a highly competitive personal and work environment of tremendous daily pressure in the area of Anaesthesiology, it is necessary to invest in practices which conduct to a balance of challenges, expectations and self-fulfilment, in order to improve the quality of life (QoL) of professionals.

The goal of this study is to evaluate the QoL of residents and attendings of Anaesthesiology in Portugal and to identify the factors that influence it.

\section{Materials and Methods}

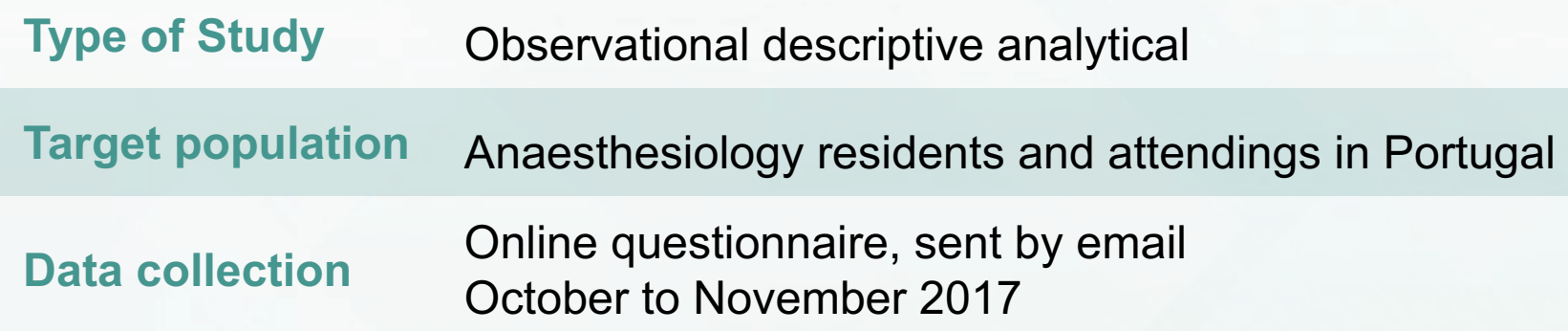
general QoL assessment and 24 items organized in 4 domains: physical, psychological, social and environmental

Scores were converted into a $0-100 \%$ scale Higher scores indicating better QoL

Descriptive: frequencies, percentages and medians

Statistical

analysis

Analytical: t-test, ANOVA, Spearman correlation $\alpha=0.05 ;$ SPSS $24.0^{\circledR}$

Results

\begin{tabular}{|c|}
\hline 110 physicians \\
\hline 56 residents \\
54 attendings
\end{tabular}

\begin{tabular}{c}
\hline Median age \\
$\mathbf{3 1}$ years old \\
$(\min 26-\max 62)$ \\
\hline
\end{tabular}

Median time as
attending
$\mathbf{9 , 5}$ years

Table 1. Sample characterization

\begin{tabular}{|llll} 
Variable & \multicolumn{1}{c}{$\mathbf{N}(\%)$} & \multicolumn{1}{c}{ Variable } & \multicolumn{1}{c}{$\mathbf{N}(\%)$} \\
\hline Gender & $\begin{array}{l}\text { Feminine } 80(72,7 \%) \\
\text { Masculine 30 }(27,3 \%)\end{array}$ & $\begin{array}{l}\text { University } \\
\text { hospital }\end{array}$ & $\mathbf{6 7}(\mathbf{6 0}, 9 \%)$ \\
\hline $\begin{array}{l}\text { Marital } \\
\text { status }\end{array}$ & $\begin{array}{l}\text { Single 48 }(43,6 \%) \\
\text { Married 58 }(52,7 \%) \\
\text { Divorced 4 (3,6\%) }\end{array}$ & $\begin{array}{l}\text { Country } \\
\text { region }\end{array}$ & $\begin{array}{l}\text { North 32 }(29,1 \%) \\
\text { Centre 48 }(43,6 \%) \\
\text { South 28 }(25,5 \%)\end{array}$ \\
$\begin{array}{l}\text { With } \\
\text { children }\end{array}$ & $41(37,3 \%)$ & & \\
\hline
\end{tabular}

Chart 1. Hospital activity

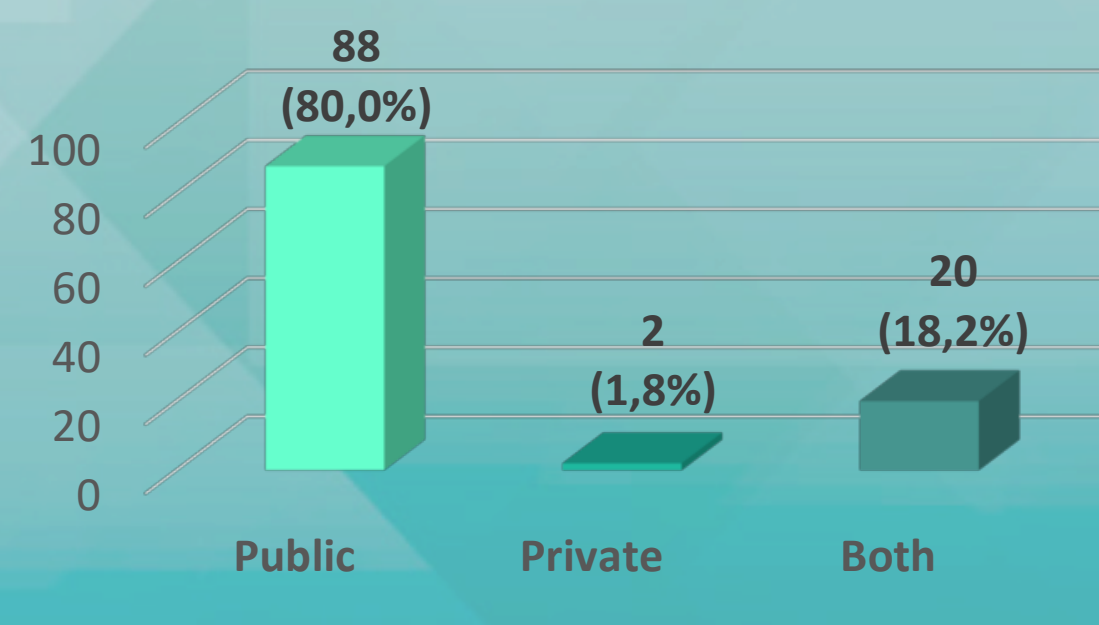

Chart 2. Weekly workload (hours)

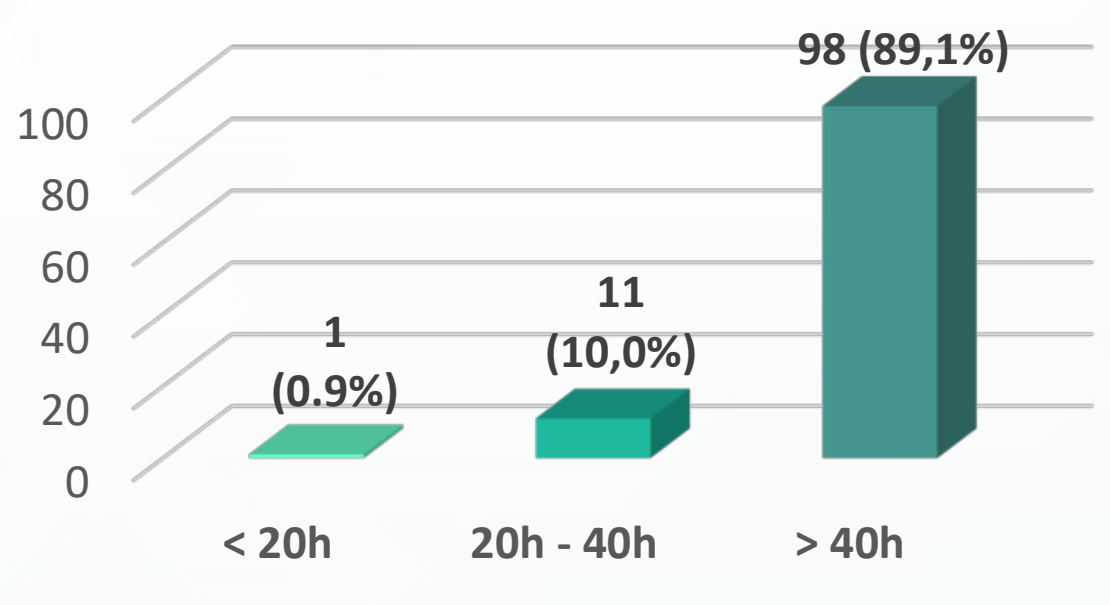

Chart 3. Other variables

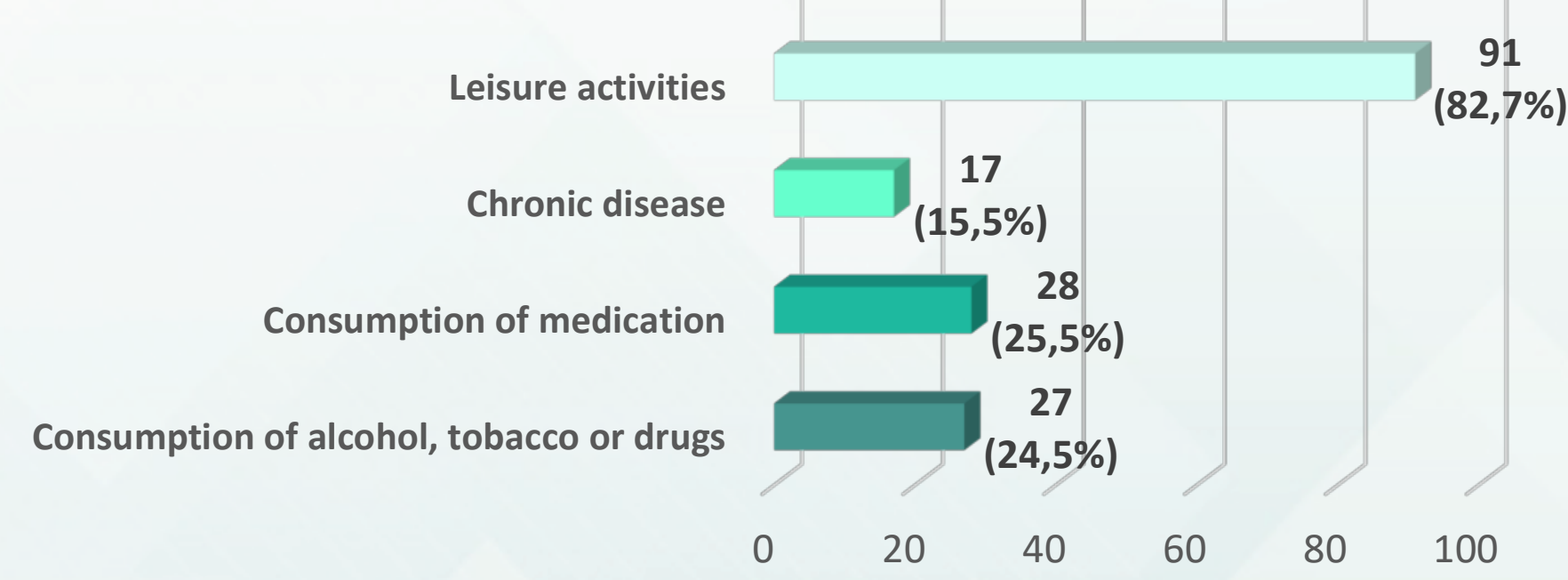

Table 2. General QoL and other domains; comparison between residents and attendings

\begin{tabular}{lc|ccc}
\multicolumn{1}{c|}{ Domains } & Total & Residents & Attendings & $\boldsymbol{p}$ \\
\hline General QoL & $71,4 \pm 19,0$ & $73,9 \pm 18,2$ & $68,7 \pm 19,7$ & 0,158 \\
\hline Physical & $78,3 \pm 12,6$ & $78,9 \pm 11,8$ & $77,7 \pm 13,5$ & 0,623 \\
\hline Psychological & $77,0 \pm 13,5$ & $77,6 \pm 13,9$ & $76,5 \pm 13,2$ & 0,661 \\
\hline Social & $73,8 \pm 17,1$ & $76,0 \pm 14,9$ & $71,4 \pm 18,9$ & 0,160 \\
\hline Environment & $71,2 \pm 13,2$ & $73,4 \pm 12,5$ & $69,0 \pm 13,8$ & 0,081
\end{tabular}

Table 3. Factors influencing general QoL and other domains

\begin{tabular}{llcc}
\multicolumn{1}{c}{ Domains } & Leisure (+) & Chronic disease $(-)$ & Medication $(-)$ \\
General QoL & $p=0,005^{*}$ & $p=0,003^{*}$ & $p=0,010^{*}$ \\
Physical & $p=0,047^{*}$ & $p<0,001^{*}$ & $p=0,006^{*}$ \\
Psychological & $p=0,001^{*}$ & $p=0,016^{*}$ & $p=0,026^{*}$ \\
Social & $p=0,004^{*}$ & $p=0,016^{*}$ & $p=0,033^{*}$ \\
Environment & $p=0,007^{*}$ & $p=0,067$ & $p=0,300$
\end{tabular}

\section{Discussion and Conclusions}

Physicians obtained average scores higher than $65 \%$ in the general QoL and in the 4 domains of the questionnaire. We did not find statistically significant differences in the responses given by residents when compared to attendings.

The domain with the best score was the physical domain and the worst was the environment domain.

Chronic disease and habitual consumption of medication had a negative impact on general QoL and on the physical, psychological and social domains.

The practice of leisure activities was the only factor that positively influenced overall QoL and all other domains. 